\title{
Footpoint excitation of standing acoustic waves in coronal loops ${ }^{\star}$
}

\author{
Y. Taroyan ${ }^{1}$, R. Erdélyi ${ }^{2}$, J. G. Doyle ${ }^{1}$, and S. J. Bradshaw ${ }^{3}$ \\ 1 Armagh Observatory, College Hill, Armagh BT61 9DG, N. Ireland \\ e-mail: yat@arm.ac.uk \\ 2 Space and Atmosphere Research Center, Department of Applied Mathematics, University of Sheffield, The Hicks Building, \\ Hounsfield Road, Sheffield S3 7RH, UK \\ 3 Space and Atmospheric Physics Group, Blackett Laboratory, Imperial College London, Prince Consort Road, \\ London SW7 2BZ, UK
}

Received 31 January 2005 / Accepted 24 March 2005

\begin{abstract}
A new theoretical model for the study of slow standing sausage mode oscillations in hot $(T>6 \mathrm{MK})$ active region coronal loops is presented. These oscillations are observed by the SUMER spectrometer on board the SoHO satellite. The model contains the transition region and the upper chromosphere which enables us to study the entire process of hot loop oscillations from the impulsive footpoint excitation phase to the rapid damping phase. It is shown that standing acoustic waves can be excited by an impulsive heat deposition at the chromospheric footpoint of a loop if the duration of the pulse matches the fundamental mode period. The pulse is immediately followed by a standing wave consistent with the SUMER observations in hot loops. The amount of released energy determines the oscillation amplitude. The combined effects of thermal conduction and radiation on the behaviour of the standing acoustic waves in hot gravitationally stratified loops are investigated. In addition to damping, these effects lead to downflows which are superimposed on the oscillations. The implications of the results in coronal seismology are discussed.
\end{abstract}

Key words. Sun: atmosphere - Sun: activity - Sun: oscillations - hydrodynamics

\section{Introduction}

In the past few years observations by high-resolution space imaging telescopes and spectrometers have confirmed the view that the solar atmosphere has a very complex structure in which a great variety of magnetohydrodynamic (MHD) waves are supported. MHD waves are an important diagnostic tool for the determination of the physical parameters of the medium in which they propagate (MHD seismology). MHD waves are also natural carriers of energy and represent a possible source for heating of the solar coronal plasma and for solar wind acceleration. Solar coronal structures support both propagating and standing MHD waves. Propagating slow magnetoacoustic waves have been detected in coronal plumes (Ofman et al. 1997, 1999) and near the footpoints of coronal loops (Berghmans \& Clette 1999; De Moortel et al. 2000). Williams et al. (2001) have revealed fast magnetoacoustic waves propagating along a loop. Among the standing fast MHD waves observed in coronal loops are the global kink (Aschwanden et al. 1999; Nakariakov et al. 1999) and sausage mode oscillations (Nakariakov et al. 2003).

* Appendices $\mathrm{A}$ and $\mathrm{B}$ are only available in electronic form at http://www . edpsciences.org
Oscillations interpreted as standing slow magnetoacoustic waves have been recently detected in hot $(T>6 \mathrm{MK})$ loops with the SUMER spectrometer on board the SoHO satellite (Kliem et al. 2002; Wang et al. 2002, 2003). The observed periods are between 7-31 min. The outward propagating slow waves observed near the footpoints of coronal loops by EIT and TRACE can be continuously present for several hours. The oscillations observed by SUMER are most likely excited impulsively, as evidenced by the presence of large initial Doppler shifts and impulsive profiles of intensity and line width. The initial pulses with amplitudes up to $200 \mathrm{~km} \mathrm{~s}^{-1}$ are followed by rapidly damped oscillations. The background sound speed in hot loops is $\approx 300-400 \mathrm{~km} \mathrm{~s}^{-1}$ and, therefore, the oscillations can be highly nonlinear. The damping times are between 5.7 to $36.8 \mathrm{~min}$, i.e., they are proportional to the periods. These oscillations usually have longer periods and larger amplitudes than the standing kink mode oscillations in cool loops observed by TRACE. For comparison, the displacement amplitudes are about 4-5 times larger. Also, the scaling of the damping time with the period is different. Unlike the transverse loop oscillations the SUMER hot loop oscillations are usually not associated with large GOES flares. They are believed to be excited in the lower parts of the atmosphere near one of the footpoints. 
These facts suggest that the oscillations observed by SUMER belong to a different type. The following arguments favour the interpretation in terms of slow standing (or acoustic) waves. The period of slow standing waves in a coronal loop can be expressed in terms of the tube (or sound) speed and the loop length (Roberts et al. 1984). The SUMER data show that the phase speeds derived from the periods and loop lengths agree well with the actual sound speed. Whether a slow mode wave is a propagating or standing wave can be inferred from the phase relationship between velocity and intensity: the phase difference is $1 / 4$ period for standing waves and zero for propagating waves. The fact that the intensity fluctuation lags behind the Doppler shifts by a quarter period confirms that the oscillations observed by SUMER are slow standing modes.

The cause of the rapid decay of the oscillations observed by SUMER has attracted the attention of several authors. The transverse standing loop oscillations observed by TRACE are believed to be damped by resonant absorption (Ruderman \& Roberts 2002; Goossens et al. 2002) or phase mixing (Ofman $\&$ Aschwanden 2002) due to enhanced shear viscosity or resistivity. The mechanism responsible for the damping of transverse waves is different from that acting on slow (predominantly longitudinal) waves. In the latter case the main dissipation mechanisms are thermal conduction along magnetic field lines and compressive viscosity. Ofman \& Wang (2002) modelled the damping of slow standing waves with typically observed solar parameters. They find that the damping time due to compressive viscosity is an order of magnitude longer than the damping time observed by SUMER. Because of the high temperature of the loops, the large thermal conduction leads to rapid damping of the oscillations. The scaling of the damping time with period agrees well with the scaling derived by Wang et al. (2003) from the observations. De Moortel \& Hood (2003) showed that in the linear limit the damping time is somewhat longer compared to the observations. Mendoza-Briceño et al. (2004) showed that the inclusion of gravitational stratification results in a further 10-20\% reduction of the damping time. The isothermal 1D loop models used in the above mentioned studies were enough to explain the rapid damping of the slow standing mode oscillations and showed that the decay time is mainly governed by the thermal conduction timescale.

The transverse kink mode oscillations are excited when a loop is hit by nearby erupting filaments, flares and/or coronal mass ejections. The process of the excitation of standing acoustic waves is less clear. It has been demonstrated numerically that the second harmonic of a standing acoustic wave is readily excited in flaring loops regardless of the location of the heat deposition in the loop (Nakariakov et al. 2004; Tsiklauri et al. 2004). However, many of the events observed by SUMER have periods corresponding to the period of a fundamental mode standing acoustic wave. The footpoint brightenings seen in SXT images and the upward moving EUV emission along the loop near the brightening footpoint suggest that the slowstanding waves in hot loops seen by SUMER could be excited by pressure disturbances associated with the injection of hot plasma at the oscillating loop's footpoint. (Wang et al. 2003) It has been speculated that the brightening and the plasma injection near the footpoint of an oscillating loop could be due to a sudden energy release caused by the process of magnetic reconnection (Sarro et al. 1999; Wang et al. 2003). Alternatively, the heat deposition could be due to nonlinear Alfvén waves (Moriyasu et al. 2004).

Recently there have been reports on possible correlations between the oscillations observed in the lower and upper regions of the solar atmosphere (O'Shea et al 2002; De Pontieu et al. 2003, 2005). In many cases the observations indicate that the oscillations in the corona are excited at chromospheric or even photospheric heights (e.g. De Pontieu et al. 2004). In the present paper we attempt to further develop these ideas by investigating the possibility of the excitation of standing acoustic waves in coronal loops at their chromospheric footpoints as suggested by the observations. The plasma temperature inside the loop undergoes steep variation from the footpoint to the apex. Therefore, the loop models with constant background temperature are insufficient for the theoretical investigation of the excitation process of standing loop oscillations.

In the present work a $1 \mathrm{D}$ gravitationally stratified loop model with an inhomogeneous temperature profile is applied to the study of standing acoustic waves in coronal loops. The temperature range covers the upper chromosphere, the transition region and the corona. This allows us to study the entire oscillation process - from the impulsive footpoint excitation phase to the rapid damping phase. It is shown how the fundamental standing slow waves can be excited by an impulsive energy release at the chromospheric footpoint of a loop. The numerical analysis is complemented by an analytical study. An important parameter on which the existence of the oscillations depends is the duration of the heat deposition. The effects of thermal conduction and radiative cooling are examined.

\section{Formulation of the problem}

The longitudinal waves can be described by a 1D semicircular loop model in which the only coordinate is the distance $s$ along the loop. The magnetic field guides the motion although it does not appear explicitly in the governing equations. The acoustic wave motion is governed by the nonlinear hydrodynamic (HD) equations which can be represented in the conservative form:

$\frac{\partial \rho}{\partial t}+\frac{\partial \rho v}{\partial s}=0$

$\frac{\partial \rho v}{\partial t}+\frac{\partial \rho v^{2}}{\partial s}=-\frac{\partial p}{\partial s}+\rho g_{\|}$,

$\frac{\partial e}{\partial t}+\frac{\partial}{\partial s}[(e+p) v]=\rho v g_{\|}+\mathcal{S}$,

where

$e=\frac{p}{\gamma-1}+\frac{\rho v^{2}}{2}$

$p=\frac{R}{\tilde{\mu}} \rho T$.

Equations (1)-(3) are solved for the density $\rho$, the total energy density $e$ and the momentum density $\rho v$, where $v$ is the $s$ component of the velocity along the loop and

$g_{\|}=-g \cos \left(\frac{\pi s}{L}\right)$ 
is the gravitational acceleration along the loop. In Eqs. (1)-(6) $\mathcal{S}, R, \tilde{\mu}, \gamma$ and $L$ represent the net effect of sources and sinks of energy, the gas constant, the mean molecular weight, the adiabatic index and the total length of the loop, respectively. It has been mentioned in the introduction that several mechanisms could be responsible for the generation of standing acoustic waves in coronal loops. From a mathematical point of view the problem is reduced to appropriately selecting the initialboundary conditions and the term $\mathcal{S}$ on the righ-hand side of Eq. (3) in such a way that standing acoustic waves are set up in a loop with given parameter values.

\section{Homogeneous loops}

We first consider the simple case of an ideal homogeneous loop in which all background quantities are constants. Assuming that the perturbations are small we may linearise and reduce the governing equations to the wave equation

$\frac{\partial^{2} v}{\partial t^{2}}=c_{\mathrm{s}}^{2} \frac{\partial^{2} v}{\partial s^{2}}, \quad t>0, \quad 0<s<L$,

where $c_{\mathrm{s}}$ is the constant sound speed inside the loop. In the case of reflecting boundaries Eq. (7) has standing acoustic wave solutions which can be represented in the form

$v(t, s)=\cos \left(\frac{\pi k c_{\mathrm{s}} t}{L}\right) \sin \left(\frac{\pi k s}{L}\right)$.

Each mode is characterised by a harmonic number $k=1,2, \ldots$, a period $P=2 L /\left(k c_{\mathrm{s}}\right)$ and an initial velocity profile

$v(0, s)=\sin \left(\frac{\pi k s}{L}\right)$.

An energy release at the apex will drive flows in opposite directions which may lead to the initial profile (9) with $k=2$, i.e., to the generation of the second harmonic. This idea has been tested numerically and applied to the study of flaring loops (Nakariakov et al. 2004). However, Eq. (9) gives no clear hints as to how the fundamental mode $(k=1)$ can be excited in a coronal loop.

Standing acoustic waves of the form

$v(t, s)=\sin \left(\frac{(2 k-1) \pi c_{\mathrm{s}} t}{2 L}\right) \cos \left(\frac{(2 k-1) \pi s}{2 L}\right)$

can be generated by a continuous sinusoidal driver at the footpoint $s=0$. The period of the fundamental mode is $P=4 L / c_{\mathrm{s}}$. Meanwhile, the observations indicate that the period of the fundamental mode is twice shorter. This supports the idea that standing acoustic waves in coronal loops observed by SUMER are not likely to be driven continuously at the footpoints of the loops.

Finally, we examine the excitation of standing acoustic waves by a single pulse at the loop footpoint. Plasma can be impulsively injected into the loop as a result of a microflare or another process which releases energy at the footpoint of the loop. We impose the following initial and boundary conditions on the solution of the wave Eq. (7):

$v(0, s)=\frac{\partial}{\partial t} v(0, s)=0$, $v(t, 0)= \begin{cases}A \sin ^{2}\left(\frac{\pi t}{P}\right), & 0 \leq t \leq P, \quad v(t, L)=0, \\ 0, & t>P,\end{cases}$

where $A$ and $P=2 L / c_{\mathrm{s}}$ are the velocity amplitude and the duration of the plasma injection, respectively. The solution of the problem (7), (11), (12) is

$$
\begin{aligned}
v(t, s)= & A H\left(t-\frac{s}{c_{\mathrm{s}}}\right) \sin ^{2}\left[\frac{\pi c_{\mathrm{s}}}{2 L}\left(t-\frac{s}{c_{\mathrm{s}}}\right)\right] \\
& -A H\left(t-\frac{2 L-s}{c_{\mathrm{s}}}\right) \sin ^{2}\left[\frac{\pi c_{\mathrm{s}}}{2 L}\left(t+\frac{s}{c_{\mathrm{s}}}\right)\right],
\end{aligned}
$$

where $H$ is the Heaviside step function. Equation (13) is derived using the useful technique of Laplace transforms which has been applied to the study of other problems in solar physics (see, e.g., Berghmans \& De Bruyne 1995; Sutman et al. 1998). The details of the derivation are presented in Appendix A.

For $t>2 L / c_{\mathrm{s}}$ Eq. (13) is reduced to

$v(t, s)=-A \sin \left(\frac{\pi c_{\mathrm{s}} t}{L}\right) \sin \left(\frac{\pi s}{L}\right)$

The injection of plasma at the footpoint of a homogeneous loop with a velocity profile given by Eq. (12) leads to the formation of a fundamental mode standing acoustic wave (Eq. (14)) after a time period of $t=P$, where $P=2 L / c_{\mathrm{s}}$ is the fundamental mode period. In other words, the standing wave is set up immediately after the plasma injection.

It should be mentioned that the choice of the boundary conditions (12) is important. The mathematical form of the initial pulse determines whether a standing wave is formed or not. For example, if Eq. (12) is replaced by

$v(t, 0)= \begin{cases}A \sin \left(\frac{\pi t}{P}\right), & 0 \leq t \leq P, \\ 0, & t>P(t, L)=0,\end{cases}$

then the stading wave solution (14) is replaced by

$v(t, s)=-\frac{A}{\pi} \sum_{k=1}^{\infty} \frac{8}{4 k^{2}-1} \sin \left(\frac{\pi k c_{\mathrm{s}} t}{L}\right) \sin \left(\frac{\pi k s}{L}\right), t>P$

(see Appendix B). Equation (16) represents a wave which propagates forth and back inside the loop despite having a period $P=2 L / c_{\mathrm{s}}$. The dotted line in Fig. 1 shows the variation of the corresponding wave crest inside the loop. The duration of the heat input $P$ is also essential for the existence of the standing waves. In the above analysis the value of $P$ is fixed at $P=2 L / c_{\mathrm{s}}$. Let us now assume that the boundary conditions are given by Eq. (12), where $P=L / c_{\mathrm{s}}$ or $P=4 L / c_{\mathrm{s}}$. For $P=L / c_{\mathrm{s}}$ and $t>P$ the solution can be represented in the form

$$
\begin{aligned}
v(t, s)= & -\frac{A}{2} \sin \left(\frac{2 \pi c_{\mathrm{s}} t}{L}\right) \sin \left(\frac{2 \pi s}{L}\right)+\frac{A}{\pi} \sum_{k=1}^{\infty} \frac{8}{\left(4 k^{2}-1\right)(2 k-3)} \\
& \times \cos \left(\frac{\pi(2 k-1) c_{\mathrm{s}} t}{L}\right) \sin \left(\frac{\pi(2 k-1) s}{L}\right)
\end{aligned}
$$

(see Appendix B). The solution in Eq. (17) has a period of $2 L / c_{\mathrm{s}}$. However, it represents a wave which propagates forth and back inside the loop being reflected at the boundaries. The dashed line in Fig. 1 represents the corresponding variation of 


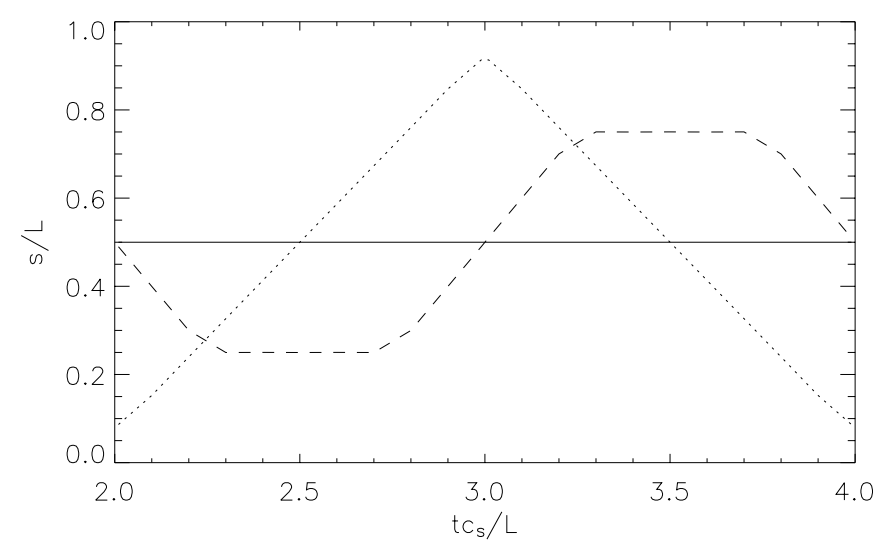

Fig. 1. The position of the wave crest inside the loop as a periodic function of time. Distance is normalised with respect to the loop length $L$ and time is normalised with respect to $L / c_{\mathrm{s}}$. The dotted line is for Eq. (16) and the dashed line is for Eq. (17). The solid line represents the fixed crest position of the standing wave solution (14).

the position of the wave crest inside the loop. For $P=4 L / c_{\mathrm{s}}$ the solution is represented by the formula

$$
\begin{aligned}
v(t, s)= & A H\left(t-\frac{s}{c_{\mathrm{s}}}\right) \sin ^{2}\left[\frac{\pi c_{\mathrm{s}}}{4 L}\left(t-\frac{s}{c_{\mathrm{s}}}\right)\right] \\
& +A H\left(t-\frac{2 L+s}{c_{\mathrm{s}}}\right) \sin ^{2}\left[\frac{\pi c_{\mathrm{s}}}{4 L}\left(t-\frac{2 L+s}{c_{\mathrm{s}}}\right)\right] \\
& -A H\left(t-\frac{2 L-s}{c_{\mathrm{s}}}\right) \sin ^{2}\left[\frac{\pi c_{\mathrm{s}}}{4 L}\left(t-\frac{2 L-s}{c_{\mathrm{s}}}\right)\right] \\
& -A H\left(t-\frac{4 L-s}{c_{\mathrm{s}}}\right) \sin ^{2}\left[\frac{\pi c_{\mathrm{s}}}{4 L}\left(t-\frac{4 L-s}{c_{\mathrm{s}}}\right)\right]
\end{aligned}
$$

(see Appendix A). It is straightforward to show that the lefthand side of Eq. (18) equals zero when $t>4 L / c_{\mathrm{s}}$, i.e., the motion vanishes together with the pulse.

\section{Inhomogeneous loops}

The results of the previous section can be applied to the analysis of standing waves in inhomogeneous loops. All initial equilibrium quantities in an inhomogeneous loop such as temperature, density, pressure and gravity along the loop are variable functions of the coordinate $s$. The process of excitation of the standing acoustic waves and their subsequent damping is examined by solving the fully nonlinear governing Eqs. (1)-(5). The sources and the sinks of energy are represented by the term $\mathcal{S}$ in Eq. (3). In order to distinguish the effects of inhomogeneity from the effects of dissipation on the standing acoustic waves, we consider two separate cases in the following subsections. In the first case all dissipative terms in Eq. (3) are switched off, whereas in the second the effects of radiative and thermal losses on the oscillations in inhomogeneous loops are examined.

\subsection{No dissipation}

The absence of dissipation means that the term $\mathcal{S}$ in Eq. (3) contains no sinks of energy. We choose a loop length of $150 \mathrm{Mm}$ and an apex temperature of $6 \mathrm{MK}$. These values are typical for the oscillating loops observed by SUMER. The initial temperature profile along the loop is shown in Fig. 2a. The loop is symmetric with respect to the apex and contains a $1.5 \mathrm{Mm}$ long and $20000 \mathrm{~K}$ hot chromosphere on each side. Other quantities such as density and pressure are derived by solving the hydrostatic analogues of Eqs. (1)-(5).

The flow-through boundary conditions are implemented at the boundaries of the loop, i.e., the value of a given convected quantity is extrapolated to a grid point just outside the boundary. In order to make the flow-through boundary conditions compatible with the requirement of hydrostatic pressure balance, we introduce regions of suppressed gravity near the left and right boundaries which are well beyond the domain of interest. The grid is nonuniform with a high spatial resolution around the transition region. The nonlinear governing Eqs. (1)-(3) are solved for the conservative variables $\rho, \rho v$ and $e$ using the total variation diminishing (TVD) scheme with a Woodward limiter (Tóth 1996). Test runs show that the evolution of the initial state develops flows which are small (less than $100 \mathrm{~m} \mathrm{~s}^{-1}$ ) and should not have a significant influence on the results of the simulations. We may therefore assume that the initial loop atmosphere is in hydrostatic equilibrium.

The initial state is disturbed by a release of energy at the bottom of the transition region near the left footpoint. The energy release is represented by the term $\mathcal{S}$ on the right-hand side of Eq. (3). Based on the results of the preceding section we choose the following functional form for the transient heat input:

$\mathcal{S}=h \equiv \begin{cases}h_{0} \sin ^{2}\left(\frac{\pi t}{P}\right) \exp \left(-\frac{s-s_{0}}{s_{\mathrm{h}}}\right), & 0 \leq t \leq P \\ 0, & t>P\end{cases}$

where the index $h_{0}$ is the maximum heat input, $s_{0}$ and $s_{\mathrm{h}}$ are the location of maximum heating and the heating scale length. The maximum heat input is fixed at the top of the upper chromosphere (bottom of the transition region), $s_{0}=1.5 \times 10^{8} \mathrm{~cm}$ $(1.5 \mathrm{Mm})$. The heating scale length is $s_{\mathrm{h}}=10^{8} \mathrm{~cm}(1 \mathrm{Mm})$. The duration of the heat input is $P=2 L / c_{\mathrm{s}}=806 \mathrm{~s}$, where $c_{\mathrm{s}}$ is the sound speed at the apex. For $h_{0}=10^{-3} \mathrm{erg} \mathrm{cm}^{-3} \mathrm{~s}^{-1}$ a fundamental mode standing acoustic wave with an amplitude of about $1 \mathrm{~km} \mathrm{~s}^{-1}$ is set up, i.e., the results of the previous section remain valid when inhomogeneity is included. Nonlinear effects become important with increasing $h$. Figure 3 shows the evolution of the velocity profile for $h_{0}=1.2 \times$ $10^{-2} \mathrm{erg} \mathrm{cm}^{-3} \mathrm{~s}^{-1}$. The oscillation is no longer a purely symmetric wave which is due to the deformation of the initially symmetric temperature profile of the loop (Fig. 2b). However, it still has a well-defined period of $806 \mathrm{~s}$ corresponding to the fundamental mode, nodes at the footpoints and an antinode near the apex. There is a slight damping caused by the wave leakage into the chromosphere which, however, cannot account for the observed rapid damping. Figure $2 b$ shows that the transition region is pushed up by about $5 \mathrm{Mm}$ in response to the energy release at the footpoint. 

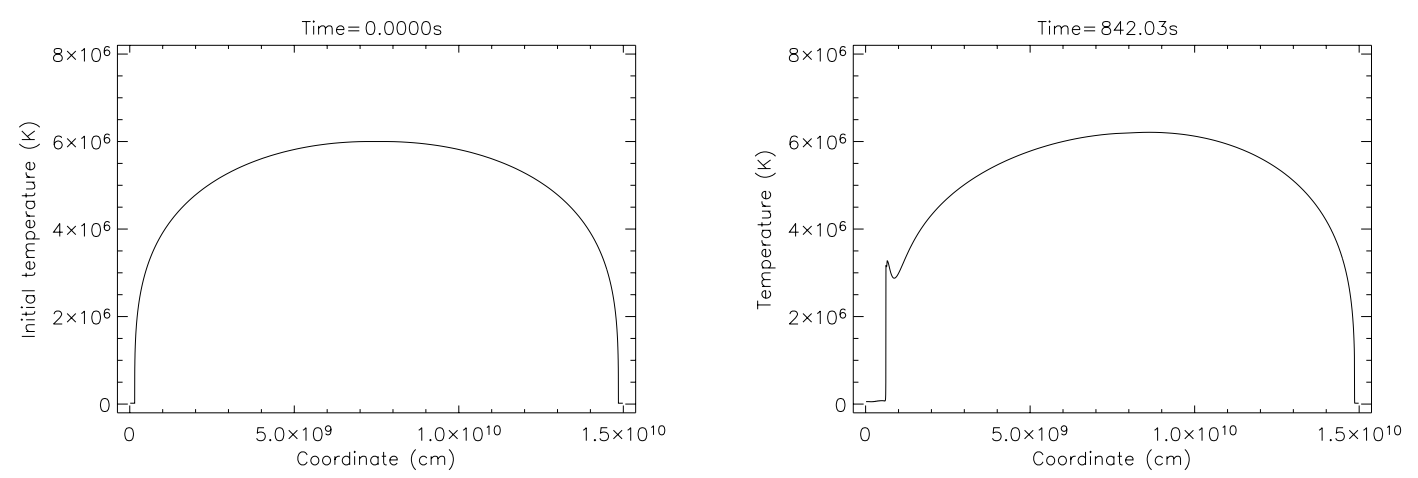

Fig. 2. a) The initial temperature profile of the loop. The chromospheric part of the loop is $1.5 \mathrm{Mm}$ long on each side and has a constant temperature of $20000 \mathrm{~K}$. The temperature increases through the narrow transition region and reaches its maximum value of $6 \mathrm{MK}$ at the loop apex; b) the initial loop temperature profile is deformed by a pulse at the left footpoint.

\subsection{Dissipative effects}

The effects of thermal and radiative losses are readily incorporated into the model through the term $\mathcal{S}$ on the right-hand side of Eq. (3):

$\mathcal{S}=\mathcal{H}+h-\frac{\partial F_{\mathrm{c}}}{\partial s}-\mathcal{L}_{r}$

Here $\mathcal{H}$ is the background heating required to balance the losses and $h$ is the transient heating defined in Eq. (19); $F_{\mathrm{c}}$ is the conductive flux along the magnetic field given by

$F_{\mathrm{c}}=-\varkappa T^{5 / 2} \frac{\partial T}{\partial s}$

where $x=10^{-6} \mathrm{erg} \mathrm{s}^{-1} \mathrm{~K}^{-1} \mathrm{~cm}^{-1}$ is the coefficient of thermal conduction along the field; the term $\mathcal{L}_{\mathrm{r}}=n^{2} \Lambda(T)$, where $n$ is the number density, corresponds to optically thin radiative losses. The chromosphere is mostly optically thick and so does not radiate as strongly as the overlying atmospheric layers. The radiative loss function $\Lambda(T)$ is smoothly decreased to zero over a very narrow temperature interval in regions where the temperature approaches the chromospheric temperature. So, what we have in the boundary region between the lower transition region and the chromosphere is a radiative loss function that will naturally and physically be a steep function of the temperature (see, e.g., Klimchuk et al. 1987; Antiochos et al 1999; Spadaroet al. 2003; Bradshaw \& Mason 2003). Furtermore, the scale length of the temperature interval over which radiation is decreased to zero is extremely small when compared with the scale length of the excitations that are generated at the bottom of the transition region, and so has a negligible effect on the results.

The conductive time step required for numerical stability is very small in an inhomogeneous hot loop. This computational problem is partly overcome by using an adaptive regridding scheme for the integration of the time-dependent hydrodynamic Eqs. (1)-(3). The conductive flux is set to zero at the footpoints of the loop. The full details of the HYDRAD code are described in Bradshaw \& Mason (2003).

It is assumed that the background heating $\mathcal{H}$ is uniform. The hydrostatic equations of pressure and energy (which now contains the thermal and radiative loss functions) are then integrated to derive the initial atmosphere. The resulting temperature profile is very similar to the one shown in Fig. 2a: the $1.5 \mathrm{Mm}$ thick chromosphere has a constant temperature of $20000 \mathrm{~K}$ and the loop reaches a peak temperature of $6 \mathrm{MK}$ at its apex. The uniform heating required to maintain this atmosphere is $\mathcal{H}=6.2 \times 10^{-3} \mathrm{erg} \mathrm{cm}^{-3} \mathrm{~s}^{-1}$. The total loop length is $150 \mathrm{Mm}$.

The initial atmosphere is disturbed by a heating pulse $h$ defined in Eq. (19), where the values of the parameters $s_{0}$ and $s_{\mathrm{h}}$ are left unchanged. The results of the previous subsection have shown that the maximum heat input required for the excitation of a fundamental mode with a velocity amplitude of $13 \mathrm{~km} \mathrm{~s}^{-1}$ is about $h_{0}=1.2 \times 10^{-2} \mathrm{erg} \mathrm{cm}^{-3} \mathrm{~s}^{-1}$. The heating and the losses add extra inertia to the loop: the amount of heat needed to excite an oscillation similar to the one shown in Fig. 3 is an order of magnitude larger compared to the heat input required in the dissipationless case. For the dissipative loop simulations we set $h_{0}=0.7 \mathrm{erg} \mathrm{cm}^{-3} \mathrm{~s}^{-1}$ and $P=780 \mathrm{~s}$. Figure 4 displays the evolution of the velocity profile through its consecutive amplitude peaks. The velocity amplitude reaches its first peak of about $50 \mathrm{~km} \mathrm{~s}^{-1}$ at $t=570 \mathrm{~s}$. At this stage the oscillation is not yet fully formed (dotted line in Fig. 4) and it has a high degree of asymmetry with respect to the apex. Consistent with the predictions of the analytical study the velocity oscillation becomes more symmetric after about $t=780 \mathrm{~s}$ and acquires typical features of a standing fundamental mode oscillation: there are nodes near the footpoints and an antinode at the apex; the oscillation has a well-defined period of about $780 \mathrm{~s}$ which is close to the analytically predicted period of the fundamental mode $P=2 L / c_{\mathrm{s}}$. Compared to the dissipationless case the oscillation has a larger initial amplitude and a lower degree of asymmetry. It is also rapidly damped. The damping time (i.e., the time over which the amplitude decreases by a factor of $e$ ) is proportional to the period which is in agreement with the results of Ofman \& Wang (2002).

Another new feature introduced by the dissipation are the downflows towards the footpoints which persist in the presence of the large amplitude velocity oscillations resulting in a slight asymmetry (Fig. 4). To find out the cause of the downflows superimposed on the fundamental mode standing acoustic wave 

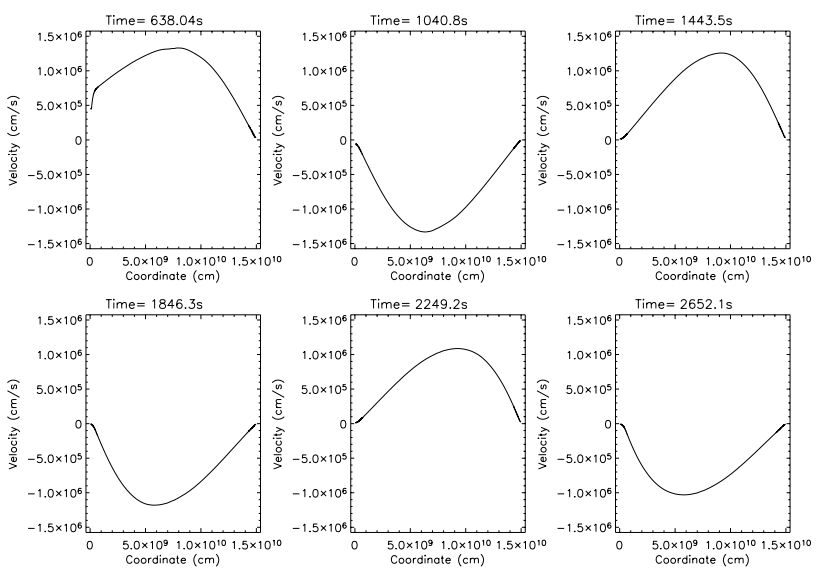

Fig. 3. The evolution of the velocity profile in response to an initial pulse. The snapshots taken at different times correspond to the consecutive peaks in the velocity amplitude.

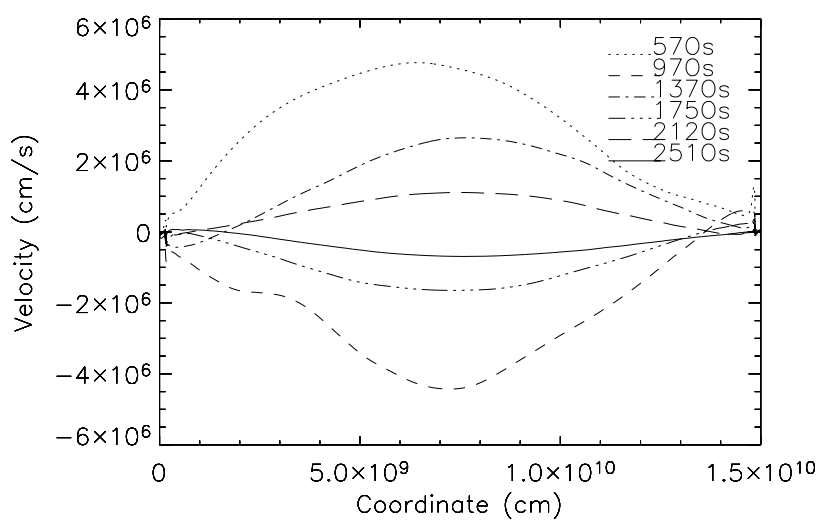

Fig. 4. The evolution of the velocity profile in response to the heat pulse at the bottom of the transition region. The different lines correspond to times when the velocity amplitude reaches its consecutive peaks.

we examine the evolution of density, temperature and conductive flux (Fig. 5).

Firstly, the temperature plot in Fig. 5 shows that the transition region has become more rigid compared to the dissipationless case (see Fig. 2b) despite the larger amount of heat deposited at the footpoint. This feature allows the velocity oscillation to remain nearly symmetric even when the amplitudes are very large (Fig. 4). The heat deposition at the footpoint ejects material from the dense chromosphere up into the corona and increases the density in the coronal part of the loop (the dotted density line in Fig. 5). The dense loop begins to cool down because of increased radiation. Indeed, the temperature plot in Fig. 5 shows that except the dotted line $(t=570 \mathrm{~s})$ all other lines at subsequent times are below the solid line which corresponds to the initial temperature profile. The temperature also starts to oscillate together with the velocity. Figure 5 shows that when $t=970 \mathrm{~s}$ the conductive flux near the right footpoint (dashed line) is below its original value (solid line) because of the reduced temperature. As a result, there is not enough conduction from above to balance the cooling by radiation below the point where the divergence of the conductive flux becomes negative. A downflow is generated towards the right footpoint
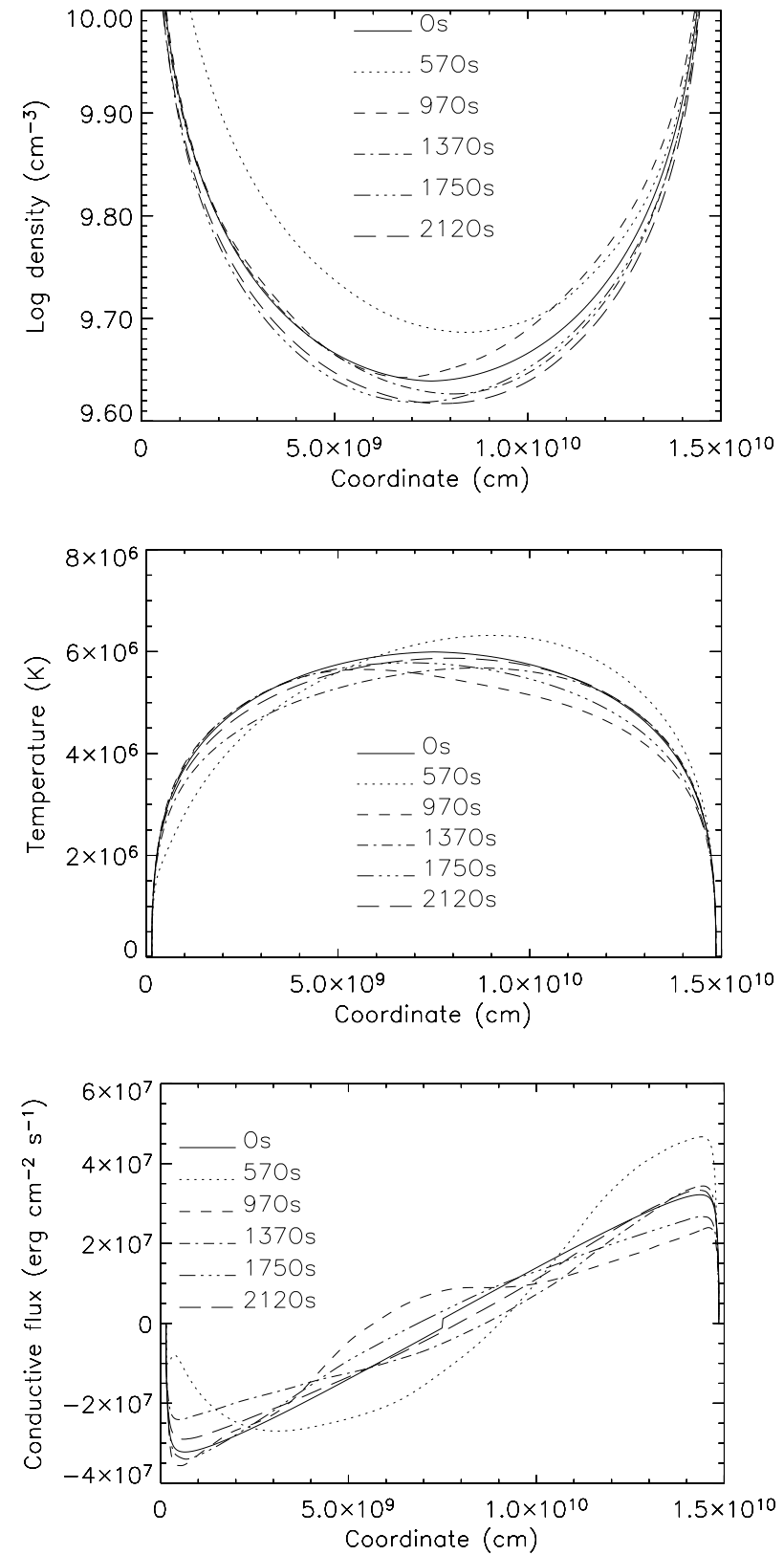

Fig. 5. The evolution of density, temperature and conductive flux. All parameters are the same as in Fig. 4.

to compensate the heat deficit. The downflow is opposite to the flow generated by the oscillation. The downflow towards the left footpoint is generated by the same mechanism.

After about $t=1370 \mathrm{~s}$ the density profile in the coronal part of the loop is reduced below the original solid line due to the persistent nature of the downflows (Fig. 5). The heating exceeds the losses and the original temperature profile of the loop is restored. The downflows vanish together with the oscillation.

As an implication in coronal seismology, the periods and the amplitudes of the standing waves could be used to estimate the duration and the amount of heat released at the footpoints of the loops. The duration of the heat release is equal to the period of the fundamental mode $P$. The location of maximum heat input $s_{0}$ determines the wave amplitude. In the present paper the footpoint excitation of acoustic waves is examined and $s_{0}$ is 


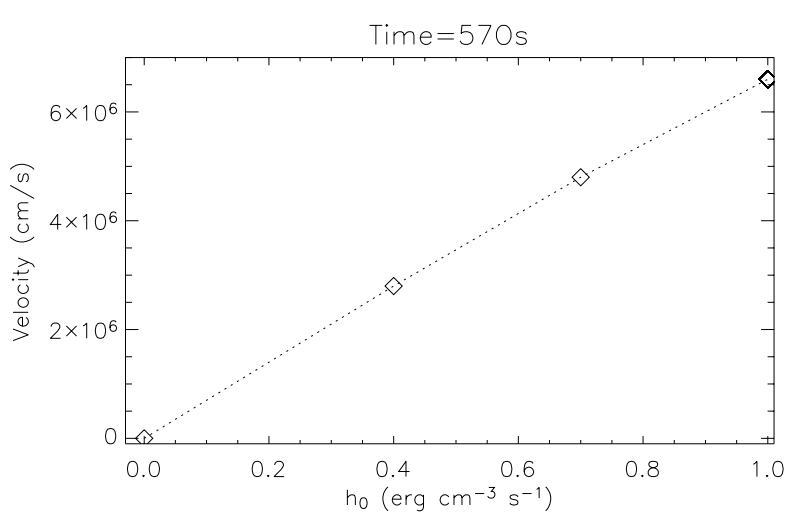

Fig. 6. The velocity maximum at $t=570 \mathrm{~s}$ as a function of the maximum heat input $h_{0}$. All other parameters are the same as in Fig. 4.

fixed at the top of the chromosphere. The amplitudes of the excited oscillations are smaller if the heat is released in the lower parts of the chromosphere. Therefore, the wave amplitude provides a lower estimate for the amount of heat released at the chromospheric footpoints of the loops. The dependence of the maximum velocity on the maximum heat input $h_{0}$ is plotted in Fig. 6. The velocity reaches its maximum at about $t=570 \mathrm{~s}$. Figure 6 shows that the velocity maximum increases as the ammount of heat deposited at the loop footpoint increases.

Additional simulations show that the main features presented in the above analysis remain valid when the loop parameters such as the maximum heat input, the loop length or the loop temperature are changed.

\section{Conclusions}

We have investigated the footpoint excitation of standing acoustic waves in coronal loops. The case of a homogeneous loop is treated analytically. It is shown that a fundamental mode standing acoustic wave can be excited by a single pulse at the footpoint if the duration of the pulse matches the period of the fundamental mode. The standing wave is set up immediately after the deposition of heat. This theoretical result is consistent with the SUMER observations and, therefore, the presented results could explain the excitation of oscillations in hot loops (Wang et al. 2003). The amplitude of the oscillation is proportional to the pulse amplitude. The results and conclusions of the linear analysis remain valid for an inhomoheneous stratified loop. The oscillations are initiated by a heating pulse at the chromospheric footpoint of the loop. The oscillations become more and more asymmetric and the velocity amplitude increases when the heat input is increased. The inclusion of dissipation has several effects: the heat input required to excite standing waves with similar amplitudes increases by a factor of 10; the transition region does no longer freely move in response to the heat pulse so that standing waves with much larger amplitudes can be excited; the oscillations are rapidly damped mainly due to the thermal conduction and the damping time is proportional to the period (this is in agreement with the conclusions of previous studies by Wang et al. 2003; Ofman \& Wang 2002; Mendoza-Briceño et al. 2004); background downflows towards the footpoints are superimposed on the oscillations. The mechanism responsible for the downflows is explained.

The presented results have two important implications in coronal seismology: it is shown that the duration of the heat release is equal to the period of the fundamental mode $P$; the velocity amplitude maximum provides a lower estimate for the amount of heat released at the chromospheric footpoint of the loop.

The SUMER oscillations are mainly detected in the Fe XIX and Fe XXI spectral lines which have formation temperature higher than $6 \mathrm{MK}$. One reason for this preference of high temperatures could be the following: the oscillation looses its standing wave pattern when the velocity amplitude becomes too large approaching the background sound speed: the downflows dominate the oscillation and it can no longer be identified as a standing wave. The sound speed is higher in hot loops and therefore the standing waves in hot loops can have large enough amplitudes to be observed. However, the fact that the Doppler signal is weak does not necessarily mean that the standing waves cannot exist in cooler loops.

Acknowledgements. Research at the Armagh Observatory is grantaided by the N. Ireland Dept. of Culture, Arts and Leisure. Y.T. is grateful to PPARC for grant PPA/G/S/2002/00020. The simulations were run on the Armagh cluster, funded by a PRTLI research grant for Grid-enabled Computational Physics of Natural Phenomena (Cosmogrid), and on White Rose Grid (Titania node at Sheffield University). R.E. acknowledges M. Kéray for patient encouragement and the financial support from NSF, Hungary (grant No. OTKA TO43741). The authors also thank the anonymous referee for the constructive remarks.

\section{References}

Antiochos, S. K., MacNeice, P. J., Spicer, D. S., \& Klimchuk, J. A. 1999, ApJ, 512, 985

Aschwanden, M. J., Fletcher, L., Schrijver, C. J., \& Alexander, D. 1999, ApJ, 520, 880

Berghmans, D., \& Clette, F. 1999, Sol. Phys., 186, 207

Berghmans, D., \& De Bruyne, P. 1995, ApJ, 453, 495

Bradshaw, S. J., \& Mason, H. E. 2003, A\&A, 407, 1127

De Moortel, I., \& Hood, A. 2003, A\&A, 408, 755

De Moortel, I., Ireland, J., \& Walsh, R. W. 2000, A\&A, 355, L23

De Pontieu, B., Erdélyi, R., \& De Moortel, I. 2005, ApJ, submitted

De Pontieu, B., Erdélyi, R., \& De Wijn, A. G. 2003, ApJ, 595, L63

De Pontieu, B., Erdélyi, R., \& James, S. P. 2004, Nature, 430, 546

Goossens, M., Andries, J., \& Aschwanden, M. J. 2002, A\&A, 394, L39

Kliem, B., Dammasch, I. E., Curdt, W., \& Wilhelm, K. 2002, ApJ, 568, L61

Klimchuk, J. A., Antiochos, S. K., \& Mariska, J. T. 1987, ApJ, 320, 409

Mendoza-Briceño, C. A., Erdélyi, R., \& Sigalotti, L. Di G. 2004, ApJ, 605,493

Moriyasu, S., Kudoh, T., Yokoyama, T., \& Shibata, K. 2004, ApJ, 601, L107 
Nakariakov, V. M., Ofman, L., DeLuca, E. E., Roberts, B., \& Davila, Spadaro, D., Lanza, A. F., Lanzafame, A. C., et al. 2003, ApJ, 582, J. M. 1999, Science, 285, 862 486

Nakariakov, V. M., Melnikov, V. F., \& Reznikova, V. E. 2003, A\&A, Sutman, G., Musielak, Z. E., \& Ulmschneider, P. 1998, A\&A, 340, 412, L7 556

Nakariakov, V. M., Tsiklauri, D., Kelly, A., Arber, T. D., \& Ruderman, M. S., \& Roberts, B. 2002, ApJ, 577, 475 Aschwanden, M. J. 2004, A\&A, 414, L25

Ofman, L., \& Aschwanden, M. J. 2002, ApJ, 576, L153

Ofman, L., \& Wang, T. J. 2002, ApJ, 580, L85

Tóth, G. 1996, Astrophys. Lett. Comm., 34, 245

Tsiklauri, D., Nakariakov, V. M., Arber, T., \& Aschwanden, M. J. 2004, A\&A, 422, 351

Ofman, L., Rokoli, M., Poletto, G., Noci, C., \& Kohl, J. L. 1997, ApJ 491, L111

Wang, T. J., Solanki, S. K., Curdt, W., Innes, D. E., \& Dammasch, I. E. 2002, ApJ, 574, L101

Ofman, L., Nakariakov, V. M., \& DeForest, C. E. 1999, ApJ, 514, 441

O’Shea, E., Muglach, K., \& Fleck, B. 2002, A\&A, 387, 642

Roberts, B., Edwin, P. M., \& Benz, A. O. 1984, ApJ, 279, 857

Wang, T. J., Solanki, S. K., Curdt, W., et al. 2003, A\&A, 406, 1105

Williams, D. R., Phillips, K. J. H., Rudawy, P., et al. 2001, MNRAS, 326,428

Sarro, L. M., Erdélyi, R., Doyle, J. G., \& Perez, M. E. 1999, A\&A, 351, 721 
Y. Taroyan et al.: Footpoint excitation of standing acoustic waves in coronal loops, Online Material p 1

\section{Online Material}




\section{Appendix A: Derivation of formulae (13) and (18)}

Let $\mathcal{V}(\sigma, s)$ be the Laplace transform of $v(t, s)$ :

$\mathcal{V}(\sigma, s)=\int_{0}^{\infty} v(t, s) \exp (-\sigma t) \mathrm{d} t$

The initial conditions (11) imply that the Laplace transform of Eq. (7) has the form

$\frac{\partial^{2} \mathcal{V}}{\partial s^{2}}=\frac{\sigma^{2}}{c_{\mathrm{s}}^{2}} \mathcal{V}$

The general solution of Eq. (A.2) is

$\mathcal{V}(\sigma, s)=a(\sigma) \exp \left(-\frac{\sigma}{c_{\mathrm{s}}} s\right)+b(\sigma) \exp \left(\frac{\sigma}{c_{\mathrm{s}}} s\right)$.

The boundary conditions (12) are transformed into

$\mathcal{V}(\sigma, 0)=\frac{2 A \pi^{2}[1-\exp (-P \sigma)]}{P^{2} \sigma\left[\sigma^{2}+\left(\frac{2 \pi}{P}\right)^{2}\right]}, \quad \mathcal{V}(\sigma, L)=0$.

Applying the boundary conditions (A.4) with $P=2 L / c_{\mathrm{s}}$ to the solution (A.3), one finds

$$
\begin{aligned}
\mathcal{V}(\sigma, s)= & \frac{2 A \pi^{2}}{P^{2} \sigma\left(\sigma^{2}+\left(\frac{2 \pi}{P}\right)^{2}\right)} \\
& \times\left[\exp \left(-\frac{\sigma}{c_{\mathrm{s}}} s\right)-\exp \left(-\frac{\sigma}{c_{\mathrm{s}}}(2 L-s)\right)\right] .
\end{aligned}
$$

Taking the inverse Laplace transform of Eq. (A.5) and using the second shifting theorem, we obtain the solution (13).

The derivation of Eq. (18) consists of the same steps as those used to derive Eq. (13).

\section{Appendix B: Derivation of formulae (16) and (17)}

We start with the derivation of Eq. (16). Taking the Laplace transform of the boundary conditions (15), we have

$\mathcal{V}(\sigma, 0)=\frac{A \pi[1+\exp (-P \sigma)]}{\sigma^{2}+\left(\frac{\pi}{P}\right)^{2}}, \quad \mathcal{V}(\sigma, L)=0$,

where $P=2 L / c_{\mathrm{s}}$. The boundary condition (B.1) combined with Eq. (A.3) leads to the solution

$$
\begin{aligned}
\mathcal{V}(\sigma, s)= & \frac{A \pi[1+\exp (-P \sigma)]}{P\left[\sigma^{2}+\left(\frac{\pi}{P}\right)^{2}\right][1-\exp (-P \sigma)]} \\
& \times\left[\exp \left(\frac{\sigma s}{c_{\mathrm{s}}}\right)-\exp \left(\frac{\sigma(2 L-s)}{c_{\mathrm{s}}}\right)\right] .
\end{aligned}
$$

The inverse Laplace transform of Eq. (B.2) is given by the Bromwich integral

$$
v(t, s)=\frac{1}{2 \pi i} \int_{\alpha-i \infty}^{\alpha-i \infty} \exp (\sigma t) \mathcal{V}(\sigma, s) \mathrm{d} \sigma .
$$

The integration in Eq. (B.3) is to be performed along a line $\sigma=\alpha$ in the complex plane $\sigma$. The real number $\alpha$ is chosen so that $\sigma=\alpha$ lies to the right of all singularities but is otherwise arbitrary. The function $\mathcal{V}(\sigma, s)$ has simple poles at

$\sigma= \pm \frac{\pi i}{P} ; \quad \sigma=\sigma_{k}=\frac{2 \pi i k}{P}, k=0, \pm 1, \pm 2, \ldots$,

all of which lie on the imaginary axis. Therefore, the line $\sigma=$ $\alpha_{0}=1 / P$ lies to the right of the poles. Let $\Delta_{m}$ be a circle with centre at the origin $O$ and radius

$R_{m}=\frac{2 \pi}{P}\left(m+\frac{1}{2}\right)$

where $m$ is a positive integer. The required inverse (B.3) can be found by considering a closed contour $C_{m}$ which consists of the line from $\alpha_{0}-i \sqrt{R_{m}^{2}-\alpha_{0}^{2}}$ to $\alpha_{0}+i \sqrt{R_{m}^{2}-\alpha_{0}^{2}}$ and the arc of $\Delta_{m} \Gamma_{m}$, traversed in the positive (counterclockwise) direction:

$$
\begin{aligned}
v(t, s) & =\lim _{m \rightarrow \infty} \frac{1}{2 \pi i} \int_{\alpha_{0}-i \sqrt{R_{m}^{2}-\alpha_{0}^{2}}}^{\alpha_{0}+i \sqrt{R_{m}^{2}-\alpha_{0}^{2}}} \exp (\sigma t) \mathcal{V}(\sigma, s) \mathrm{d} \sigma \\
& =\lim _{m \rightarrow \infty} \frac{1}{2 \pi i}\left(\oint_{C_{m}}-\int_{\Gamma_{m}}\right) \exp (\sigma t) \mathcal{V}(\sigma, s) \mathrm{d} \sigma .
\end{aligned}
$$

The choice of $R_{m}$ in Eq. (B.5) ensures that the contours $C_{m}$ and $\Gamma_{m}$ do not pass through any of the poles. In general, the evaluation of the right-hand side of Eq. (B.6) is not straightforward. However, we find that when $t>P$ the integral around $\Gamma_{m}$ in Eq. (B.6) approaches zero as $m \rightarrow \infty$. This is done using the substitution $\sigma=R_{m} \exp (i \theta)$. Therefore, the Bromwich integral is reduced to

$v(t, s)=\lim _{m \rightarrow \infty} \frac{1}{2 \pi i} \oint_{\mathcal{C}_{m}} \exp (\sigma t) \mathcal{V}(\sigma, s) \mathrm{d} \sigma, \quad t>P$.

According to the residue theorem the right-hand side of Eq. (B.7) is equal to the sum of residues of the integrand $\exp (\sigma t) \mathcal{V}(\sigma, s)$ at all poles of $\mathcal{V}(\sigma, s)$. The residues at the poles $\sigma= \pm i \pi c_{\mathrm{s}} /(2 L)$ equal zero because of the presence of the factor $1+\exp (-P \sigma)$ in Eq. (B.2). The resudues at $\sigma=\sigma_{k}$, $k=0, \pm 1, \pm 2, \ldots$, are

$\lim _{\sigma \rightarrow \sigma_{k}}\left(\sigma-\sigma_{k}\right) \exp (\sigma t) \mathcal{V}(\sigma, s)$

$$
=-\frac{4 i A}{\pi\left(4 k^{2}-1\right)} \exp \left(-i \pi k \frac{c_{\mathrm{s}} t}{L}\right) \sin \left(\frac{\pi k s}{L}\right),
$$

where the limit was evaluted using L'Hospital's rule. Taking the sum of the residues, we have

$$
\begin{aligned}
v(t, s) & =-\frac{A}{\pi} \sum_{k=-\infty}^{\infty} \frac{4 i}{4 k^{2}-1} \exp \left(-i \pi k \frac{c_{\mathrm{s}} t}{L}\right) \sin \left(\frac{\pi k s}{L}\right) \\
& =-\frac{A}{\pi} \sum_{k=1}^{\infty} \frac{8}{4 k^{2}-1} \sin \left(\pi k \frac{c_{\mathrm{s}} t}{L}\right) \sin \left(\frac{\pi k s}{L}\right), \quad t>P .
\end{aligned}
$$

Finally, we present the derivation of Eq. (17). This case is treated in a manner similar to the previous case. For the sake 
Y. Taroyan et al.: Footpoint excitation of standing acoustic waves in coronal loops, Online Material p 3

of brevity we will only outline the main steps of the derivation. The Laplace transform of the boundary conditions is represented by Eq. (A.4), where $P$ now has the form $P=L / c_{\mathrm{s}}$. The solution of the problem (A.2), (A.4) is

$\mathcal{V}(\sigma, s)=\frac{4 A \pi^{2} \sinh \left[\sigma(L-s) / c_{\mathrm{s}}\right]}{P^{2} \sigma\left[\sigma^{2}+\left(\frac{2 \pi}{P}\right)^{2}\right][1+\exp (-P \sigma)]}$.

It has simple poles at

$\sigma=0, \pm \frac{2 \pi i}{P} ; \sigma=\sigma_{k}=\frac{(2 k-1) \pi i}{P}, k=0, \pm 1, \pm 2, \ldots$,

all of which are again located on the imaginary axis. We can therefore go through a similar procedure to find the required inverse $v(t, s)$. The analysis shows that the second integral in Eq. (B.6) [where $\mathcal{V}(\sigma, s)$ is now the right-hand side of Eq. (B.10)] approaches zero as $m \rightarrow \infty$ provided $t>P$. The problem is thus reduced to the evaluation of the integral in
Eq. (B.7), where $\mathcal{V}(\sigma, s)$ is given by Eq. (B.10). The residue at $\sigma=0$ is zero due to the presence of the sinh function in Eq. (B.10). The residues at $\sigma= \pm 2 \pi i / P$ are

$$
\begin{aligned}
\lim _{\sigma \rightarrow \pm 2 \pi i / P}\left(\sigma \mp \frac{2 \pi i}{P}\right) \exp (\sigma t) \mathcal{V}(\sigma, s) \\
= \pm \frac{i A}{4} \exp \left(\frac{2 i \pi c_{\mathrm{s}} t}{L}\right) \sin \left(\frac{2 \pi s}{L}\right) .
\end{aligned}
$$

The residues at $\sigma=\sigma_{k}, k=0, \pm 1, \pm 2, \ldots$, where $\sigma_{k}$ is defined in Eq. (B.11), are evaluated using L'Hospital's rule:

$$
\begin{array}{r}
\lim _{\sigma \rightarrow \sigma_{k}}\left(\sigma-\sigma_{k}\right) \exp (\sigma t) \mathcal{V}(\sigma, s)=\frac{4 A}{\pi\left(4 k^{2}-1\right)(2 k-3)} \\
\times \exp \left(\frac{i \pi(2 k-1) c_{\mathrm{s}} t}{L}\right) \sin \left(\frac{\pi(2 k-1) s}{L}\right) .
\end{array}
$$

Taking the sum of all residues in Eqs. (B.12) and (B.13), we arrive at Eq. (17). 